\title{
FOODOMICS: PREFACE
}

Alejandro Cifuentes

The impressive analytical developments achieved at the end of the twentieth century have made possible the sequencing of nearly the whole human genome at the beginning of the twenty-first century, opening the so-called postgenomic era. These advances have made feasible analytical instruments and methodological developments that were unthinkable a few decades ago. These impressive developments have traditionally found their first application in the biotechnological or biochemical field many times linked to pharmaceutical, medical, or clinical needs. The huge amount of money allocated to these fields of research is logically an additional push to be considered when selecting the area in which a new analytical method can be probed, a good way to compensate the efforts behind any innovative analytical development. As a result, biotech, pharmaceutical, and clinical related industries have usually been the first targets for analytical chemists and instrumentation companies. This has left food analysis overshadowed and connected to the use of more traditional analytical approaches. Nowadays, boundaries among the different research fields are becoming more and more diffuse giving rise to impressive possibilities in the emerging interdisciplinary areas, for example, health and food. As a result, researchers in food science and nutrition are being pushed to move from classical methodologies to more advanced strategies usually borrowing methods well established in medical, pharmacological, and/or biotechnology research. This trend has generated the emergence of new areas of research for which a new terminology is required. In this context, our group defined a few years ago Foodomics, as a discipline that studies the food and nutrition domains through the application of advanced omics technologies to improve consumer's well-being, health, and confidence. The main idea behind the use of this newterm has been not only to use it as a flag of the newtimes for food analysis but also to highlight that the investigation into traditional and new problems in food analysis in the postgenomic era can find exciting opportunities and new answers through the use of genomics, transcriptomics, epigenetics, proteomics, and metabolomics tools. Indeed, Foodomics is opening a new and unexpected land still wild, still unexplored, to a new generation of researchers who, using the everyday more powerful omics technologies, can find original search possibilities and innovative answers to crucial questions not only related to food science but also related to its complex links with our health.

The interest of the scientific community in modern food analysis and Foodomics, and the different trends in this hot area of research are well documented in the 20 chapters that compose this volume on "Foodomics: Advanced Mass Spectrometry in Modern Food Science and Nutrition", the first book devoted to this new discipline in which the authors present their advanced perspective of the topic. Namely, in the first chapter the principles of Foodomics are presented, the next five chapters (chapter 2 to 6) are devoted to proteomics applications in Foodomics, including a description of modern instruments and methods for proteomics, proteomic-based techniques for food science and food allergens characterization, examination of antioxidant food supplements using advanced proteomics methods and proteomics 
in nutritional systems biology. The next two chapters (chapters 7 and 8) are devoted to the description of advanced MS-based methodologies to study transgenic foods development and characterization and the microbial metabolome. The following nine chapters (chapters 9 to 17) are devoted to metabolomics developments in Foodomics with special emphasis on the possibilities of MSbased metabolomics in nutrition and health research, for food safety, quality, and traceability, the investigations on future personalized nutrition, the study of the effect of the diet on acute and endurance exercise, the investigation on diet-related diseases, and the study on how Foodomics impact optimal nutrition or can provide crucial information on micronutrients (the case of folates), phenolic compounds as functional ingredients, and lipids (lipidomics). The following two chapters (chapters 18 and 19) present the main principles of Green Foodomics and the use of chemometrics in mass spectrometry and Foodomics. The last chapter of the book is devoted to the description of the possibilities of systems biology in food and nutrition research.

As editor of this book devoted to "Foodomics: Advanced Mass Spectrometry in Modern Food Science and Nutrition", I would like to thank all the authors for their suitable contributions, Dom Desiderio for inviting me to prepare this piece of work, Michael Leventhal for his help and support, and to those in the John Wiley \& Sons team who contributed their effort to the preparation of this volume. 